\title{
Applying Operating System Principles to SDN Controller Design
}

\author{
Matthew Monaco, Oliver Michel, Eric Keller \\ University of Colorado at Boulder \\ ffirst.last $\}$ ecolorado.edu
}

\begin{abstract}
Rather than creating yet another network controller which provides a framework in a specific (potentially new) programming language and runs as a monolithic application, in this paper we extend an existing operating system and leverage its software ecosystem in order to serve as a practical SDN controller. This paper introduces yanc, a controller platform for software-defined networks which exposes the network configuration and state as a file system, enabling user and system applications to interact through standard file $\mathrm{I} / \mathrm{O}$, and to easily take advantage of the tools available on the host operating system. In yanc, network applications are separate processes, are provided by multiple sources, and may be written in any language. Applications benefit from common and powerful technologies such as the virtual file system (VFS) layer, which we leverage to layer a distributed file system on top of, and Linux namespaces, which we use to isolate applications with different views (e.g., slices). In this paper we present the goals and design of yanc. Our initial prototype is built with the FUSE file system in user space on Linux and has been demonstrated with a simple static flow pusher application. Effectively, we are making Linux the network operating system.
\end{abstract}

\section{Categories and Subject Descriptors}

C.2.4 [Computer-Communication Networks]: Distributed Systems-Network Operating Systems

\section{General Terms}

Design, Experimentation, Management

Permission to make digital or hard copies of all or part of this work for personal or classroom use is granted without fee provided that copies are not made or distributed for profit or commercial advantage and that copies bear this notice and the full citation on the first page. To copy otherwise, to republish, to post on servers or to redistribute to lists, requires prior specific permission and/or a fee.

Hotnets '13, November 21-22, 2013, College Park, MD, USA.

Copyright 2013 ACM 978-1-4503-2596-7 ...\$10.00.

\section{INTRODUCTION}

The introduction of software-defined networks has generated tremendous buzz in the past few years as it promises to ease many of the network management headaches that have been plaguing network operators for years $[12,14]$. Software-defined networking uses a logically centralized control plane to manage a collection of packet processing and forwarding nodes in the data plane. It has been proposed that this requires an operating system for networks [16] which provides an interface to program the entire network. Applications on top of the operating system perform the various management tasks by utilizing the operating system's interface. At a high level, an OS manages the interface with hardware (network devices) and allows applications (network management tasks) to run.

Despite a useful analogy, the practical realization is that while extensible, the current SDN controllers $[16$, $3,7,24,9]$ are geared towards single, monolithic network applications where developers can write modules in the supported language using the API provided by the framework, compile the entire platform, and run as a single process. An alternate approach is to use new languages and compilers that allow programmers to specify the application with a domain specific language and run the compiled executable, still as a monolithic application [11, 21]. Among the downsides of a monolithic framework is that a bug in any part of the application (core logic, a module, etc.) can have dire consequences on the entire system.

Moreover, each of the existing controllers end up independently needing and developing a similar set of required features. This results in a fragmented effort implementing common features where the main distinguishing aspect in each case is commonly the language in which applications are allowed to be written (e.g., NOX-C++, Ryu-Python, Floodlight-Java, Nettle-Haskell, etc). Further, these controllers are missing important features like the ability to run across multiple machines (i.e., a distributed controller) - limited to a hot standby (in the case of Floodlight) or a custom integration into a particular controller (in the case of Onix [19] 
on top of NOX). Even support for the latest protocol is lacking; many have yet to move past OpenFlow 1.0 for which newer versions have been released, the latest being 1.3.1 [12] — even Floodlight, a commercially available controller, only supports 1.0 [1].

In this paper, we explore the question: Is a network operating system fundamentally that different from an operating system such that it requires a completely new (network) operating system? We argue that instead of building custom SDN controllers, we should leverage existing operating system technology in building an SDN controller. Our goal is to extend an existing operating system (Linux) and its user space software ecosystem in order to serve as a practical network OS.

\subsection{Yanc}

We present the initial design of yanc ${ }^{1}$, which effectively makes Linux the network operating system and is rooted in UNIX philosophies (§2). The yanc architecture, illustrated in Figure 1, builds off of a central abstraction used in operating systems today - the file system. With yanc, the configuration and state of the network is exposed as file I/O ( $(3)$ - allowing running application software in a variety of forms (user space process, cron job, command line utility, etc) and developing in any language. Much like modern operating systems, system services interact with the real hardware through drivers, and supporting applications can provide features such as virtualization, or supporting libraries such as topology discovery (§4). By using Linux, we can leverage the ecosystem of software that has been developed for it ( $(5)$. One special example that is made possible by building yanc into an existing operating system is that distributed file systems can be layered on top of the yanc file system to realize a distributed controller (§6). Finally, while we mostly discuss yanc in terms related to the OpenFlow protocol for ease of understanding, we believe the design of yanc, extends into more recent research, going beyond OpenFlow $(\S 7)$.

We believe that once fully implemented, yanc will enable researchers to focus on value-added applications instead of yet another network controller.

\section{GOALS}

Our goal is to extend an existing operating system (Linux) and its user space software ecosystem in order to serve as a practical network OS. Hence, our goals are similar to and extend from common Unix principles.

Applications should encompass logically distinct tasks. Each application should do one thing and do it well. For example, there should be a distinct application for each protocol the network needs to support such as DHCP, ARP, and LLDP. Furthermore, tasks

\footnotetext{
${ }^{1}$ yanc, or yet another network controller.
}

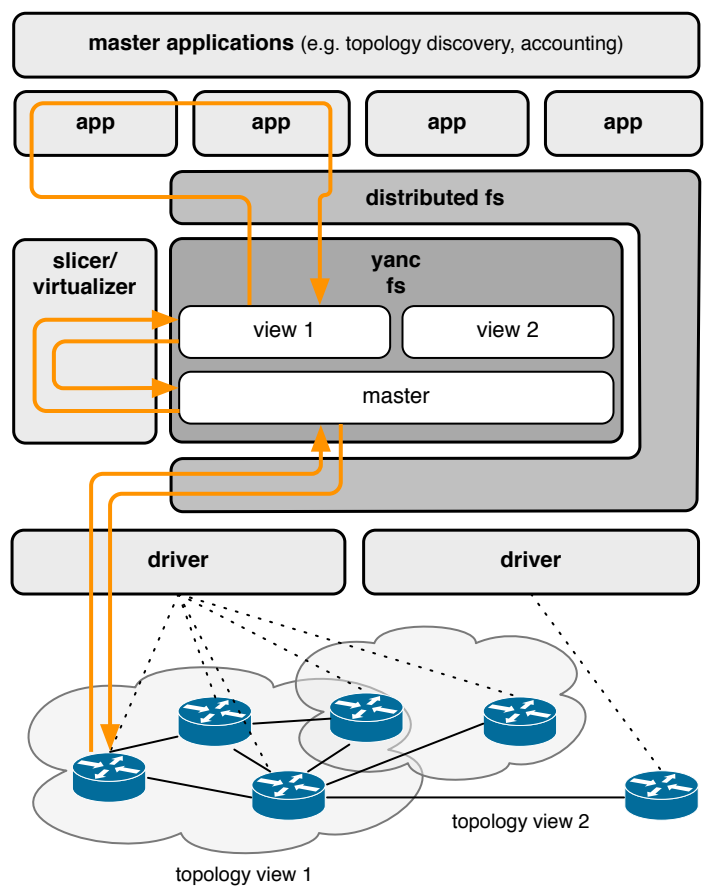

Figure 1: System Architecture

such as logging, slicing, and auditing should be packaged separately, likely with their own configuration files.

Applications may be written in any language. Rather than limiting applications to be written in the language in which the controller is implemented or has provided bindings, developers should be able to express logic in any language supported by modern operating systems. This is in contrast to the current approach where the decision for what controller to use is greatly influenced by language of choice - NOX $(\mathrm{C}++)$, Ryu (Python), Floodlight (Java), Nettle (Haskell), etc.

Applications should come from multiple sources. No one party will be an expert on all aspects of the network. Each application should assume it will be working on a network with multiple black-box applications. It is okay for one application to have specific dependencies on others, but the dependency should be on a stable API. The file system API, for example, can be used heavily for exposing and manipulating network state. For event driven applications that rely on timers and other events, Linux already provides the necessary mechanisms.

Applications should be decoupled from hardware. A device driver is the implementation of a control plane protocol (e.g., OpenFlow), or even a specific version of a protocol (e.g., OpenFlow 1.1). Drivers translate network activity for a subset of nodes to the common API supported by the network operating system. Nodes in such a system can therefore be gradually upgraded, live, to newer protocols. Another implication 
of this is that multiple protocols may be used simultaneously.

The interaction between applications should be defined by the administrator. Much of the time, multiple applications will be interested in the same network events. Which applications have priority in responding to such events should be up to the administrator of the network. Furthermore, the interaction between applications should be flexible and not limited to a linear pipeline.

Network application design should not be limitted by the controller. For some applications such as $\mathrm{ARP}$ and DHCP, a daemon processes is most appropriate as they need to be ready to handle messages at any time. Other applications, however, would be better designed as simpler programs which are run occasionally. For example an auditor might run periodically via a cron job, or LIME [18], will require the occasional reshuffling of flow entries and is best called on-demand.

Furthermore, by exposing the network through a filesystem, small shell scripts or even ad-hoc commands may be used to perform tasks on the network using existing and well-known applications in the typical coreutils suite such as cat and grep.

\section{THE YANC FILE SYSTEM}

Central to yanc is exposing network configuration and state as a file system. This decision stems from the fact that file systems are central to modern operating systems and enabling interaction with network configuration and state through file I/O enables a powerful environment for network administration. This follows from the file system abstraction providing a common interface to a wide range of hardware, system state, remote hosts, and applications. On Linux, ext4 controls access to block devices, procfs to kernel state and configuration, sysfs to hardware, and nfs to remote file systems.

In this section we describe how network configuration and state can be represented with a file system abstraction. We use aspects common to current SDN controllers and protocols (namely switches, flows, etc.). While we do have an initial prototype, some details are yet to be worked out.

\subsection{File System Layout}

At a basic level, file systems contain files which contain some information, and directories which put structure around the collection of files. The yanc file system, as shown in Figure 2, is typically mounted on /net and uses a directory structure to mimic the hierarchical nature of network configuration and state. Top level directories will represent coarse entities such as switches/ and hosts/, as well as alternate representations of the state, which we call views (discussed in $§ 4$ ).

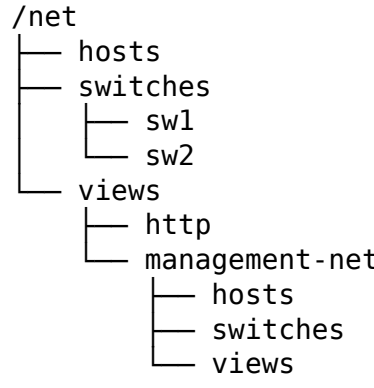

Figure 2: The yanc file system hierarchy.
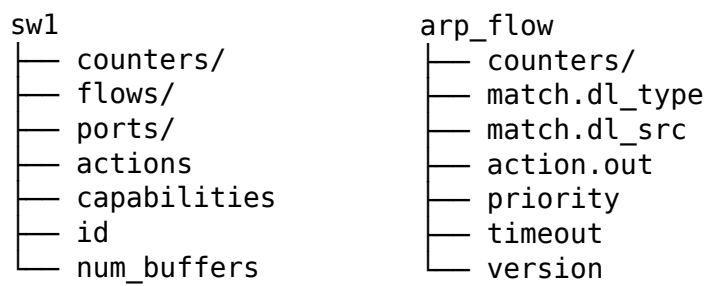

Figure 3: Partial representations of a yanc switch and flow.

The actual configuration (e.g., port status) and state (e.g., counters) are represented through a combination of files and subdirectories. For example, a port can be brought down by

\# echo $1>$ port_2/config.port_down

Importantly, with yanc, directories and files contain semantic information. With this, each directory which contains a list of objects automatically creates an object of the appropriate type on a mkdir() or create() system call. For example

\# mkdir views/new_view

will create the directory new_view, but also the hosts, switches, and views subdirectories.

\subsection{Switches}

As mentioned, a switch is a coarse entity and each will be represented with its own directory. Our current switch representation is given in Figure 3 - with files containing information about the switch (e.g., capabilities) and directories pointing to further specific configuration and state (e.g., ports). Switches can be created, deleted, and renamed with the standard file system calls (mkdir (), rmdir (), and rename (), respectively). Children of this object do not need to be removed prior to removing the object itself; in other words, the rmdir() call for switches is automatically recursive.

\subsection{Ports and Topology}

Each switch contains a set of ports. Ports are much like switches in that each is represented with a directory, and within the directory are files with information 
about the given port. This can include feature sets (e.g., what line rate is supported), state (e.g., counters), status, and configuration information (e.g., hardware address).

To represent topological information, yanc leverages symbolic links for simpler manipulation or re-organization of directory structure while still conveying the important link information (e.g., rather than parsing some topology information file). Each port contains a symbolic link named peer which may or may not exist. Pointing this link at another port is how physical links are represented. It is currently an error to point this symbolic link at anything other than a port. Link status is configured and inspected through the file config.port_status.

\subsection{Flows}

Within OpenFlow, and even many of the proposals for SDN protocols beyond OpenFlow, a flow entry is a table entry which indicates how the switch should handle traffic. As flows contain many pieces of information, each yanc flow (Figure 3) is represented by a directory. Each field that can be matched is a separate file e.g., match.nw_src. Absence of a match file implies a wildcard, and fields such as IP source take the CIDR notation. Similarly, files named action.* are used for specifying actions and files such as priority are used for setting other properties of a flow entry.

In order for multiple values to be set atomically, we currently use a version file. Applications use inotify or fanotify (§5.2) to monitor the yanc file system for new, changing, or deleted flow entries. Changes are only sent to hardware (by the drivers $(\S 4.1)$ once the version has been incremented.

The flow written by one application may need to be modified by another before being written to hardware. This can be for performance, security, or monitoring purposes. We believe that the views abstraction $(\S 4.2)$ handles the majority of these situations. However, composing networks from independent rules is still an ongoing area of research [21].

\subsection{Packet In}

Events originating on switches must be notified to applications. With yanc, events are also handled through file I/O. Specific events relating to the switch hardware status are handled by the drivers (\$4.1). For network management applications, the main event is the packetin message in OpenFlow, though any event will be handled similarly. Packet-in events are typically caused by table misses, but also by explicit rules to trigger software processing. A simple example of this is when software must respond to a new flow in a reactive network.

Our current design concurrently feeds packet-in messages to all applications interested in such events. Views
(§4.2) are used when one application needs to alter a packet-in before it is received by another (e.g., to provide a virtual switch abstraction).

To be able to receive events, each application interested in packet-in events creates a directory in the events/ subdirectory (effectively creating a private buffer). New packet-in messages appear as subdirectories within each private buffer, with files containing information about the message.

\section{SYSTEM APPLICATIONS}

The file system is effectively a mechanism to represent network configuration and state - it cannot stand on its own. With yanc, system applications form the core of necessary applications that every network management system will consist of. In this section we describe the drivers which interact with the physical switches, the applications which alter the view of the network for other applications, and the applications which discover the topological information about the network.

\subsection{Drivers}

Analogous to device drivers in operating systems, device drivers in yanc are a thin component which speaks the programming protocol supported by a collection of switches in the network. Multiple drivers for different protocols - or even protocol versions - are supported. For example the majority of switches will communicate with an OpenFlow 1.0 driver, a handful with a separate OpenFlow 1.3 driver, and others with a driver for an experimental protocol being developed. Applications will be able to inspect the files in the switches directory to know whether newer features such as quality-of-service are supported or not. With the file system as the API, supporting new protocols only requires a new driver to write new files, it does not require modifications to the core controller and interface provided to applications.

\subsection{Network Views}

Network virtualization has gone hand-in-hand with software-defined networking since SDN's inception, and has proven to be one of the 'killer applications' of SDN [10]. With yanc, we term any logical representations of an underlying network a network view. To create a new view, an application effectively interacts with two portions of the file system simultaneously - providing a translation between them. Then, any application that works off of a view will simply interact with the associated portion of the file system (which can be isolated with Linux namespaces as discussed in §5.3). With yanc, views can be stacked arbitrarily on top of one another to facilitate any logical topology and federated control required of the network.

The two main transformations in a network (today) are slicing and virtualization. A slice of a network 
is a subset of the hardware and header space across one or more switches, however the original topology is not changed [23]. Network virtualization on the other hand, provides any arbitrary transformation, such as combining multiple switches and forming a new topology through the use of overlays. These two concepts can be combined to e.g., slice traffic on port 22 out of the network, and then create a virtual single-big-switch topology.

\subsection{Discovery and Topology}

Building a topology for other applications to use is essential to the function of the control plane. As with everything in yanc, topology is represented in the file and directory layout of the file system. A topology application will handle LLDP messages for discovery and create symbolic links which connect source to destination ports. Other applications can make use of this topology to e.g., build network maps or calculate routes.

\section{LEVERAGING EXISTING SOFTWARE}

The true benefit of yanc comes from being able to use the file system in conjunction with existing (Linux) technologies and software. Here, we discuss some key components which are useful in developing a network control plane.

\subsection{Permissions, ACLs, and Attributes}

The VFS layer ( $(3)$ includes basic Unix permissions, access control lists (ACLs), and extended attributes. Modern, multi-user operating systems use permissions to control access to files and directories. Likewise, the network operating system can implement fine-grained control of network resources using permissions. For example, while individual flows can be protected for specific processes, so too can an entire switch (thus all of its flows). Making use of file permissions for network control has benefits in security, stability, and isolation.

Extended attributes are another form of metadata commonly found in file systems. In yanc, extended attributes can be used in arbitrary ways by developers. We plan on utilizing them to specify consistency requirements for various network resources (§6).

\subsection{File System Monitoring}

Applications using the yanc file system will use one of the Linux fsnotify APIs - inotify or fanotify - to monitor for changes in the network. For example, to monitor for new switches a watch can be placed on the switches directory. To monitor for a changed flow, a watch can be placed on the version file of a particular flow. This system fits in well with modern, single-eventloop designs. Furthermore, use of the *notify systems comes free, requiring no additional lines of code to the yanc file system.

\subsection{Namespaces and Control Groups}

Linux namespaces allow, on a single system, isolation of resources such as network interfaces, processes, users, and more. Control groups allow processes to be grouped in an arbitrary hierarchy for the purpose of resource management e.g., CPU, memory, and disk IO usage.

These technologies play an important role in all but the most basic of SDN deployments. They can be used to isolate subsets of the network to individual processes, Linux containers such as OpenVZ, virtual machines, and even hosts over the network. Users of these subsets are individuals, testers, federated administrators, research groups, and data-center tenants.

\subsection{Standard Utilities}

As previously mentioned, we believe that interaction with the SDN devices should be possible in many forms and not be relegated to sub-modules within a monolithic application or some interface such as a REST API. Nothing embodies this more than the rich set of command line utilities available in modern operating systems.

A quick overview of the switches in a network can be provided by:

\section{\$ Is $-1 /$ net/switches}

To list flow entries which affect ssh traffic:

\$ find /net -name tp.dst -exec grep 22

From simple one-liners to more elaborate shell scripts, these common utilities are tools that system administrators use and know, thus they should be able to be utilized in a software-defined network.

\section{DISTRIBUTED CONTROL}

When discussing SDN, papers are often careful to state that it uses a logically centralized controller, with the implication that multiple servers can be used to realize a single unified network control. To do so, each monolithic controller application must duplicate effort and implement support for it. Some example controllers include Onix, an extension of the NOX controller to integrate support for distributed controllers; ONOS [5] looks to have much in common with Onix, but open source and based on Floodlight instead of NOX; and Floodlight itself commercially supports a hot swap capability but not a fully distributed controller. In each case, they chose a distribution technology that's forced on the user, and is tied to a specific controller.

We argue that you can layer any number of distributed file systems on top of the yanc file system and arrive at a distributed SDN controller. Each distributed file system has a different implementation (centralized, peerto-peer with a DHT, etc.) with varying trade-offs. As a proof of concept, we mounted NFS on top of yanc 
and distributed computational workload among multiple machines. Other layered file systems which are likely to be useful on top of yanc are sshfs for secure, remote access and WheelFS which provides distribution and configurable consistency in a logically centralized hierarchy.

\section{BEYOND OPENFLOW}

While much of the discussion has centered around switches and to some degree, OpenFlow, yanc is not tied to either.

\subsection{Network controller, or network device?}

With a distributed file system, the lines between controller and device start to become blurred. Similar to what was envisioned with Kandoo [17], we envision network devices potentially running software that today is largely relegated to the network controller on a remote server. We would like to take advantage of vendors such as Cumulus Networks[2] and Pica8[6] which are providing devices coupled with a customizable Linux operating system.

These devices can run yanc and participate in a distributed file system rather than have a bespoke communication protocol that's dependent on how the control logic was partitioned. With yanc, when an application on another machine writes to a file representing a flow entry, that will then show up on the device (since it's a distributed file system), and the device can read it and push it into the hardware tables. Similarly, software running on a switch can make a change locally and this will be seen by remote servers. While unproven, we believe this will will be a fruitful direction to explore particularly in the quest to extend SDN to middleboxes.

\subsection{Extending to Middleboxes}

Given the limitation of OpenFlow to layers 2 through 4 with predefined processing, there has been recent research activity in extending SDN principles into middleboxes [13, 8, 22, 20].

For a middlebox with fixed funcationality, but exposing its state through a standardized protocol, a driver can be written to populate and interact with the file system and take immediate advantage of yanc- such as what's involved in the move from OpenFlow 1.0 to 1.3. This interface can be used to move the state around to elastically expand the middlebox [22]. We envision that we can use command line utilities such as cp or mv to move state around rather than custom protocols.

More interesting is the case where the device allows for custom processing. To achieve this with OpenFlow, we can either use packet-in events between device and controller or re-direct packets to custom boxes (even directly attached [15]). Using yanc coupled with a distributed file system, the device itself can run yanc, run the application software, and still be able to work under the direction of global network view.

\section{PROTOTYPE}

We have built a prototype of yanc which consists of a number of the components described in this paper. Most importantly, the core file system is implemented via the FUSE user space file system[4] (which provides for easier initial prototyping). The current yanc file system supports the introspection and manipulation of network elements, flows, ports, links, and sliced/virtual networks. Multiple tables and queues are not yet implemented. The prototype has initial but incomplete support for properly handling table misses and flow table writes in a multiprocess system.

An OpenFlow driver is implemented in $\mathrm{C}++$ and supports all of the protocol features supported by the file system. A simple "static flow pusher" shell script can be used to write flows to switches. A topology daemon is implemented in Python and maintains port-to-port symbolic links. Finally, a router daemon handles all table misses and sets up paths based on exact match through the network.

\subsection{Performance}

The file system provides a flexible namespace, access control, and a familiar programming pattern for network operators. However, it comes with some performance cost. Each fine-grained access to the file system is done through a system call - for example read(), write(), and stat() — which switches context from the application to the kernel. Complex operations such as writing flow entries to thousands of nodes will result in tens of thousands of context switches and thus a small performance impact.

To mitigate the performance overhead of working with the file system, we are implementing libyanc, a set of network-centric library calls atop a shared memory system. The library provides a fastpath for e.g., creating flow entries atomically and without any context switchings. It also allows for the efficient, zero-copy passing of bulk data — packet in buffers, for example — among applications

\section{CONCLUSION AND FUTURE WORK}

We have presented yanc, a vision of how operating system mechanisms and principles can be applied in the context of software-defined networking. Effectively, yanc realizes a network operating system which can be used in a variety of ways in order to leverage innovation in the operating system space. Thus, more focus can be put on specific control-plane-centric topics such as load balancing, congestion control, and security. Our initial prototype is only a first step toward realizing many of the aspects of yanc that we introduced in this paper. 


\section{REFERENCES}

1 Big switch networks. http://www.bigswitch.com.

[2] Cumulus networks. http://cumulusnetworks.com.

[3] Floodlight openflow controller. http://www.projectfloodlight.org.

[4] Fuse: Filesystem in userspace. http://fuse. sourceforge.net.

[5] Onos: Open network operating system. http://es.slideshare.net/umeshkrishnaswamy/ open-network-operating-system.

[6] Pica8: Open networks for software-defined networking. http://pica8.com

[7] Ryu sdn framework. http://osrg.github.io/ryu.

[8] Network functions virtualisation. In $S D N$ and OpenFlow World Congress, Oct. 2012.

[9] Z. Cai. Maestro: Achieving Scalability and Coordination in Centralized Network Control Plane. $\mathrm{PhD}$ thesis, Rice University, 2011.

[10] M. Casado, T. Koponen, R. Ramanathan, and S. Shenker. Virtualizing the network forwarding plane. In PRESTO, 2010.

[11] N. Foster, R. Harrison, M. J. Freedman, C. Monsanto, J. Rexford, A. Story, and D. Walker. Frenetic: a network programming language. In Proc. ACM SIGPLAN international conference on Functional programming (ICFP), 2011.

[12] O. N. Foundation. Openflow specification. https://www . opennetworking.org/sdn-resources/ onf-specifications/openflow.

[13] A. Gember, P. Prabhu, Z. Ghadiyali, and A. Akella. Toward software-defined middlebox networking. In Proc. Workshop on Hot Topics in Networks (HotNets), 2012.

[14] A. Greenberg, G. Hjalmtysson, D. A. Maltz, A. Myers, J. Rexford, G. Xie, H. Yan, J. Zhan, and H. Zhang. A clean slate 4d approach to network control and management. SIGCOMM Comput. Commun. Rev., 35(5):41-54, Oct. 2005.

[15] A. Greenhalgh, F. Huici, M. Hoerdt, P. Papadimitriou, M. Handley, and L. Mathy. Flow processing and the rise of commodity network hardware. ACM SIGCOMM Computer Communication Review, 39(2):20-26, 2009.

[16] N. Gude, T. Koponen, J. Pettit, B. Pfaff, M. Casado, N. McKeown, and S. Shenker. Nox: towards an operating system for networks. SIGCOMM Comput. Commun. Rev., 38(3):105-110, July 2008.

[17] S. Hassas Yeganeh and Y. Ganjali. Kandoo: a framework for efficient and scalable offloading of control applications. In Proceedings of the first workshop on Hot topics in software defined networks, pages 19-24. ACM, 2012.

[18] E. Keller, S. Ghorbani, M. Caesar, and J. Rexford. Live migration of an entire network (and its hosts). In Proceedings of the 11th ACM Workshop on Hot Topics in Networks, pages 109-114. ACM, 2012.

[19] T. Koponen, M. Casado, N. Gude, J. Stribling, L. Poutievski, M. Zhu, R. Ramanathan, Y. Iwata, H. Inoue, T. Hama, et al. Onix: A distributed control platform for large-scale production networks. In $O S D I$, volume 10, pages $1-6,2010$.

[20] J. Martins, M. Ahmed, C. Raiciu, and F. Huici. Enabling Fast, Dynamic Network Processing with ClickOS. In ACM SIGCOMM Workshop on Hot Topics in Software Defined Networking (HotSDN), 2013.

[21] C. Monsanto, J. Reich, N. Foster, J. Rexford, and
D. Walker. Composing software defined networks. In Proc. Usenix Network System Design and Implementation (NSDI), Apr 2013.

[22] S. Rajagopalan, D. Williams, H. Jamjoom, and A. Warfield. Split/merge: system support for elastic execution in virtual middleboxes. In Proc. USENIX conference on Networked Systems Design and Implementation (NSDI), 2013.

[23] R. Sherwood, G. Gibb, K.-K. Yap, G. Appenzeller, M. Casado, N. McKeown, and G. Parulkar. Can the production network be the testbed? In Proc. USENIX conference on Operating systems design and implementation (OSDI), 2010.

[24] A. Voellmy and P. Hudak. Nettle: Taking the sting out of programming network routers. In $P A D L$, pages 235-249, 2011. 